\title{
Macrophage Depletion Reduces Disease Pathology in Factor H- Dependent Immune Complex-Mediated Glomerulonephritis
}

\author{
Alexander Jacob, ${ }^{1}$ Michael Phelps, ${ }^{1}$ Shane Fraher, ${ }^{1}$ Marcos Lopez, ${ }^{1}$ Anthony Chang, ${ }^{2}$ \\ Richard J. Quigg, ${ }^{1}$ and Jessy J. Alexander $\mathbb{C D}^{1}$ \\ ${ }^{1}$ Department of Medicine, University at Buffalo, Buffalo, NY 14203, USA \\ ${ }^{2}$ Department of Pathology, University of Chicago, Chicago, IL 60637, USA \\ Correspondence should be addressed to Jessy J. Alexander; jessyale@buffalo.edu
}

Received 8 December 2021; Accepted 3 January 2022; Published 20 January 2022

Academic Editor: Baohui Xu

Copyright (c) 2022 Alexander Jacob et al. This is an open access article distributed under the Creative Commons Attribution License, which permits unrestricted use, distribution, and reproduction in any medium, provided the original work is properly cited.

Complement factor $\mathrm{H}(\mathrm{FH})$ is a key regulator of the alternative pathway of complement, in man and mouse. Earlier, our studies revealed that the absence of FH causes the C57BL6 mouse to become susceptible to chronic serum sickness (CSS) along with an increase in the renal infiltration of macrophages compared to controls. To understand if the increased recruitment of macrophages $(\mathrm{M} \phi \mathrm{s})$ to the kidney was driving inflammation and propagating injury, we examined the effect of $\mathrm{M} \phi$ depletion with clodronate in FH knockout mice with CSS. Eight-week-old FHKO mice were treated with apoferritin (4 mg/mouse) for $5 \mathrm{wks}$ and with either vehicle (PBS) or clodronate $(50 \mathrm{mg} / \mathrm{kg}$ ip, 3 times/wk for the last 3 weeks). The administration of clodronate decreased monocytes and $\mathrm{M} \phi \mathrm{s}$ in the kidneys by $>80 \%$. Kidney function assessed by BUN and albumin remained closer to normal on depletion of $\mathrm{M} \phi \mathrm{s}$. Clodronate treatment prevented the alteration in cytokines, TNF $\alpha$ and IL-6, and increase in gene expression of connective tissue growth factor (CTGF), TGF $\beta$-1, matrix metalloproteinase-9 (MMP9), fibronectin, laminin, and collagen in FHKO mice with CSS $(P<0.05)$. Clodronate treatment led to relative protection from immune complex- (IC-) mediated disease pathology during CSS as assessed by the significantly reduced glomerular pathology (GN) and extracellular matrix. Our results suggest that complement activation is one of the mechanism that regulates the macrophage landscape and thereby fibrosis. The exact mechanism remains to be deciphered. In brief, our data shows that $\mathrm{M} \phi \mathrm{s}$ play a critical role in FH-dependent ICGN and $\mathrm{M} \phi$ depletion reduces disease progression.

\section{Introduction}

Chronic kidney disease (CKD) is a global health problem; $10 \%$ of which is immune complex- (IC-) mediated and occurs in serum sickness, infections, and autoimmune diseases. [1]. The USA Renal Data System Annual Report states that individuals with CKD "are clearly at a high risk of death" with 10-fold increased incidence over three decades in which affected patients lost $70 \%$ of their lifespan. According to the NIDDK, approximately $14 \%$ of the adult population has been diagnosed with kidney disease, making it the $9^{\text {th }}$ leading cause of death [2]. The kidney is highly susceptible to complement-mediated damage especially in autoimmune and inflammatory settings $[3,4]$.
The complement system participates in innate and adaptive immune responses and provides a first line of defense against microorganisms $[5,6]$. It consists of three activation pathways, which are initiated in unique ways, and made up of more than 30 plasma and cell-associated proteins [7]. The alternative pathway is constantly on alert and is regulated by proteins such as complement factor $\mathrm{H}(\mathrm{FH})$. $\mathrm{FH}$ is a circulating protein mostly synthesized by the liver [8]. $\mathrm{FH}$-deficient $\left(\mathrm{fh}^{-1-}\right)$ mice treated with apoferritin develop chronic serum sickness (CSS) leading to diffuse proliferative glomerulonephritis (GN) within 5 weeks of treatment, while littermate $\mathrm{C} 57 \mathrm{BL} / 6$ mice that are factor $\mathrm{H}(\mathrm{FH})$ sufficient have little glomerular inflammation [9]. $\mathrm{F} 4 / 80^{+}$macrophage infiltration occurs in the kidney and is observed around IC 
TABLe 1: Primer sequences used to assess gene expression by qRT-PCR.

\begin{tabular}{lrc}
\hline Gene & \multicolumn{1}{c}{ Forward primer sequence } & Reverse primer sequence \\
\hline GAPDH, & $5^{\prime}$-TTGATGGCAACAATCTCCAC-3' & $5^{\prime}$ - CGTCCCGTAGACAAAATGGT-3' \\
Markers: & $5^{\prime}$ - GCATTAGCATGGAAGCAAAGA-3' \\
CD206 & $5^{\prime}$-CAGGAGGAGAGAGATCCGATTTA-3' \\
CD204 & $5^{\prime}$ TGGAGGAGAGAATCGAAAGCA-3 ${ }^{\prime}$ & $5^{\prime}$ - CTGGACTGACGAAATCAAGGAA-3' \\
Matrix proteins: & $5^{\prime}$ - TGCGCTTGCAGAGATTAAAA-3' \\
TGF- $\beta 1$ & $5^{\prime}$-CTTCTGGCGTGTGAGTTTCCA-3' \\
MMP9 & $5^{\prime}$-CGGAGCCCTGCATCACAAA -3' & $5^{\prime}$-AGGTAACGCCAGGAATTGTTGCTA-3' \\
Laminin, & $5^{\prime}$-ACTGCACGGTTGAAGCAAAGA-3'
\end{tabular}

ELISA: serum TNF $\alpha$ and IL-6 levels were measured by ELISA MAX ${ }^{\mathrm{TM}}$ Standard Kits (BioLegend, CA, USA) according to the manufacturer's protocols. The concentrations of IL-1B, M-CSF, and GM-CSF were measured by ELISA (R\&D Systems, UK) according to the manufacturer's instructions.

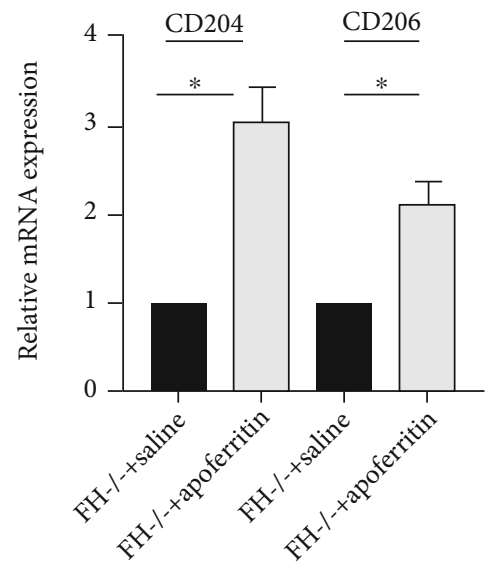

Figure 1: FH alters macrophage marker genes in the kidney of mice with FH-dependent ICGN. Real-time quantitative PCR for CD204 and CD206 using mRNA isolated from the kidneys after 5 weeks of saline or apoferritin. The expression is normalized to GAPDH ( $n=6$ /group). ${ }^{*} P<0.01$. Graphs depict fold change of genes in apoferritin-treated $\mathrm{fh}^{-1-}$ mice $(n=6)$ compared to $\mathrm{fh}^{-/ \text {- }}$ mice treated with saline $(n=6)$.

deposits in the $\mathrm{fh}^{-/-}$mice. Complement activation generates anaphylatoxins, $\mathrm{C} 3 \mathrm{a}$ and $\mathrm{C} 5 \mathrm{a}$, that participate in trafficking of $\mathrm{M} \phi$ s to the site of inflammation. Inhibition of $\mathrm{C} 5 \mathrm{a} /$ $\mathrm{C} 5 \mathrm{aR} 1$ signaling reduced the $\mathrm{M} \phi \mathrm{s}$ in the kidney [10]. In this setting, kidney injury is associated with renal infiltration but the mechanisms that may be involved in causing renal injury remains unknown.

Monocytes (Mo) generated by hematopoietic cells [11] migrate through the endothelium into the tissues, where they differentiate into $\mathrm{M} \phi \mathrm{s}$. Markers such as F4/80, CD11b, CD68, and Fc receptors can identify the $\mathrm{M} \phi \mathrm{s} . \mathrm{M} \phi \mathrm{s}$ are critically involved in renal injury, repair, and fibrosis in different experimental models of renal disease $[12,13]$. Recently, our studies demonstrated that the kidney injury in FHdependent ICGN is associated with the renal infiltration of $\mathrm{M} \phi$ s. Moreover, previous studies have demonstrated that inhibition of renal $\mathrm{M} \phi$ infiltration reduces arterial pressure and prevents glomerular injury and fibrosis in various animal models of nephropathy $[14,15]$. However, to our knowledge, the impact of $\mathrm{M} \phi$ depletion during renal injury associated with FH-dependent ICGN has not been addressed. Therefore, the present study examined the role of $\mathrm{M} \phi$ s on the renal injury associated with FH-dependent ICGN using liposome clodronate. Clodronate (clodronic acid) is a bisphosphonate that is metabolized to a nonhydrolyzable ATP analog [16]. Our experiments show that $M \phi$ s play a key role in loss of kidney function in a setting of unregulated complement activation. Therefore, reducing $\mathrm{M} \phi \mathrm{s}$ or instructing $\mathrm{M} \phi$ s to assume a repair phenotype could reduce disease pathology and can be exploited therapeutically.

\section{Materials and Methods}

2.1. Experimental Animals. The $\mathrm{fh}^{-/-}$mouse strain was generated and kindly provided by Drs. Matthew Pickering and Marina Botto (Imperial College of London) and continuously backcrossed onto the C57BL/6 strain in our laboratory for over 10 generations. Littermate FH-sufficient mice served as the controls ( $n=8$ /group) Genotyping was performed using PCR-based approaches. Mice were maintained at a temperature of $20-22^{\circ} \mathrm{C}$ in $12 \mathrm{~h}$ light $-12 \mathrm{~h}$ dark lighting cycle, in sterile, ventilated cages with food and water ad lib. The University of Chicago and University at Buffalo Institutional Animal Care and Use Committees approved all studies.

2.2. Chronic Serum Sickness Protocol. CSS was induced in 68 week $\mathrm{fH}^{-/-}$or wild-type mice with daily intraperitoneal administration of $4 \mathrm{mg}$ horse spleen apoferritin (Calzyme Laboratories) without adjuvant $[10,17,18]$ for 5 weeks. Littermate controls received intraperitoneal injections of saline vehicle contemporaneously.

2.3. Kidney Function Assessments. Blood urea nitrogen (BUN) concentrations were determined with a Beckman Autoanalyzer (Beckman Coulter, Fullerton, CA). Urinary albumin concentrations were measured with a mouse albumin ELISA kit (Bethyl Laboratories, Montgomery, TX) and normalized to urinary creatinine measured colorimetrically (Stanbio Laboratory, Boerne, TX) as described previously [19]. 


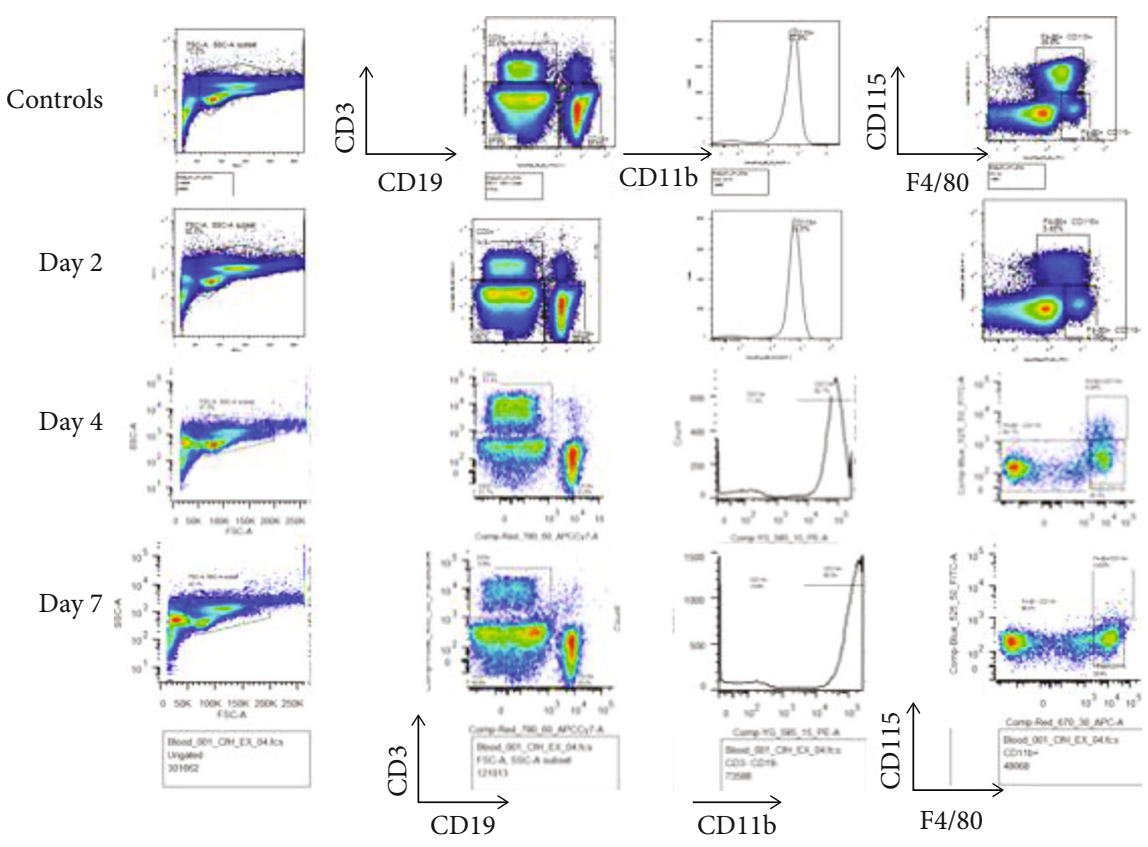

FIGURE 2: Clodronate increasingly reduces kidney macrophages in mice with FH-dependent ICGN. Given are the flow cytometry profiles of $\mathrm{F} 4 / 80+$ monocytes/M $\phi$ s on day $0,2,4$, and 7, representatively. The expression of F4/80+ cells was determined by flow cytometry. Data were analyzed using FlowJo V10 software. F4/80+ cells are significantly reduced at each time point reaching less than $10 \%$ by day 7 . Each time point $n=6$.

2.4. Histology. Kidneys were embedded in paraffin and formalin-fixed, and four-micrometer sections were generated. The sections were stained with Periodic Acid-Schiff and examined by a renal pathologist (A.C.) in a blinded manner. For each slide, proliferative glomerulonephritis, glomerulosclerosis was graded from 0 to 4 as described previously [20].

For immunofluorescence microscopy, the kidney tissue was snap-frozen in 2-methylbutane precooled in dry ice. Four-micron cryostat sections were processed for immunofluorescence (IF) staining. Sections were fixed in $4 \%$ paraformaldehyde and stained with Alexa488-conjugated anti mouse C3 (Cappel) and Alexa 555 anti-mouse IgG. A semiquantitative score of staining intensity and distribution from 0 to 4 was provided in a blinded manner as described previously [19].

2.5. $q R T-P C R$. The kidneys were harvested, and total RNA was extracted from the tissues using TRIzol reagent according to the manufacturer's instructions (Invitrogen, USA). cDNA was synthesized using SuperScript III Reverse Transcriptase (Invitrogen). $q$ RT-PCR was performed using Power SYBR Green PCR Master mix on the ABI 7700 sequence detector (Applied Biosystems). PCR amplification was performed in a total volume of $25 \mu \mathrm{l}$ containing $12.5 \mu \mathrm{l}$ of 2x TaqMan Universal PCR Master Mix (Applied Biosystems), $6.25 \mu \mathrm{l}$ of nuclease-free water, $5 \mu \mathrm{l}$ of cDNA, and $1.25 \mu \mathrm{l}$ of the appropriate primers (IDT). All qPCR primers (Table 1) were designed using the Primer3 software (Whitehead Institute for Biomedical Research) to ensure specificity and sensitivity. To quantify the levels of mRNA, we normalized expression of the target genes to glyceraldehyde-3phosphate dehydrogenase. Relative mRNA expression levels were calculated using the $2^{-\Delta \Delta \mathrm{Ct}}$ method and were normalized to the expression levels of GAPDH.

2.6. Statistics. All results are mean \pm SD from at least 6-8 mice in each group. Either an unpaired two-tailed test (data with normal distribution) or the Mann-Whitney $U$ test (data with not normal distribution) was used to compare 2 groups by GraphPad Prism 5.0. The statistical significance is expressed as follows: ${ }^{*} P<0.05 ;{ }^{* *} P<0.01$; and n.s.: not significant.

\section{Results}

We induced CSS in mice by repetitive immunization with horse spleen apoferritin. Animals generate an active antiapoferritin IgG humoral immune response and deposit $\operatorname{IgG}$ in glomeruli. Our earlier studies revealed that there is increased $\mathrm{M} \phi$ infiltration into the kidneys in this setting.

3.1. Macrophage Gene Expression Is Increased in FHDependent ICGN Kidneys. To evaluate the presence of $\mathrm{M} \phi \mathrm{s}$ in FH-dependent ICGN kidneys, qRT-PCR was performed with M2 macrophage markers, CD204 (M $\phi$ scavenger receptor 1) and CD206 ( $\mathrm{M} \phi$ mannose receptor 1) [21-24]. Expression of both genes in kidney was significantly upregulated following apoferritin treatment in FHKO mice (Figure 1) indicating increased presence of macrophages in the kidney.

3.2. Time-Course Changes by Chlodronate Treatment in Renal Macrophage Infiltration in Apoferritin-Treated FHKO Mice. To determine the effective method of reducing $\mathrm{M} \phi \mathrm{s}$ in $\mathrm{FH}$-dependent ICGN, we depleted $\mathrm{M} \phi \mathrm{s}$ in $\mathrm{fh}^{-1-}$ mice 


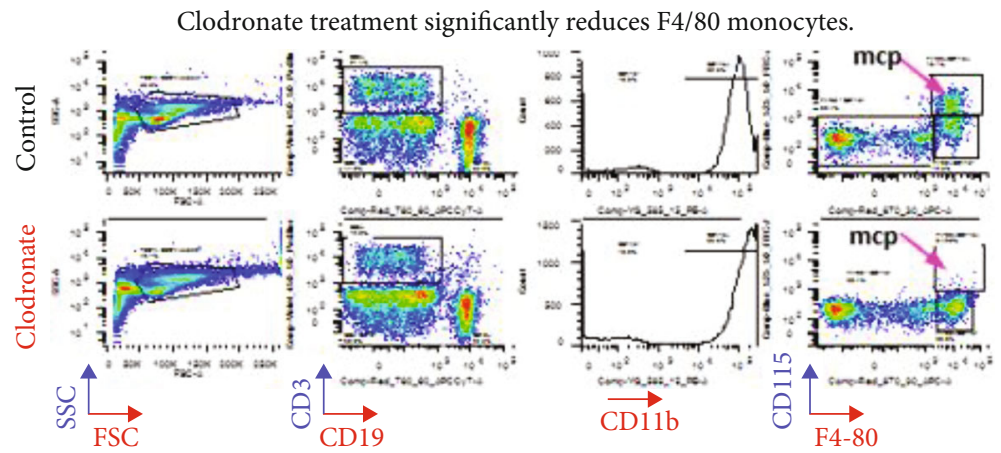

FIGURE 3: Supplemental data. Given are the flow cytometry profiles of F4/80+ monocytes from the control and clodronate-treated mice. F4/ $80+$ cells are significantly reduced reaching less than $10 \%$ by clodronate treatment. Each time point $n=6$.
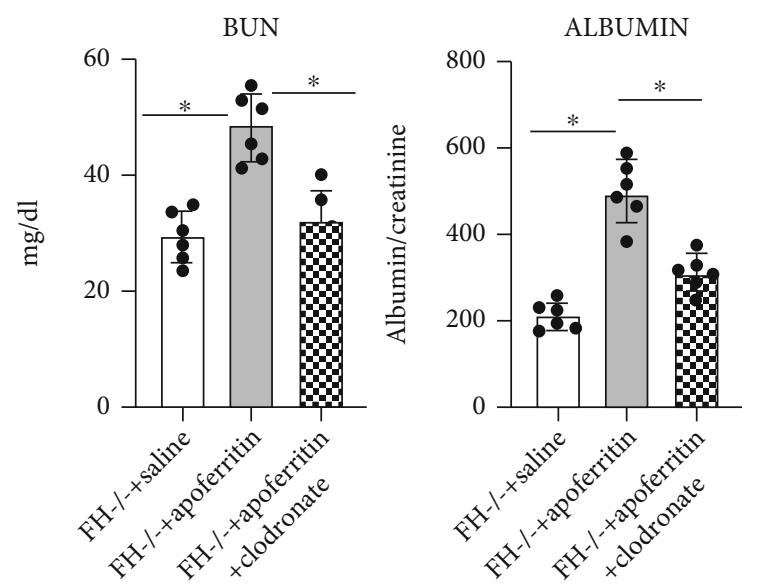

Figure 4: $\mathrm{M} \phi$ depletion prevents reduced kidney function in $\mathrm{FH}-$ dependent ICGN. Shown are final blood urea nitrogen (BUN) and albumin concentrations from $\mathrm{fH}^{-/-}$mice treated with saline (control), apoferritin (ICGN), or apoferritin+clodronate. Each circle is from an individual animal. ${ }^{*} P=0.015$ versus the control.

treated with CSS by clodronate liposome treatment. Mice were treated with clodronate liposome or control liposome every 3 days for 3 weeks. The time-course changes in renal $\mathrm{M} \phi$ infiltration in apoferritin-treated FHKO mice are shown in Figures 2 and 3. Renal $M \phi$ infiltration in the kidneys was reduced when assessed every alternate day and was a negligible presence (less than $10 \%$ similar to that observed by Kim et al. [25]) by day 7 in apoferritin-treated FHKO mice.

3.3. Macrophage Depletion Prevents Aberrant Kidney Function in FH-Mediated ICGN. The kidney function in the different mice groups was analyzed by determining serum BUN and urine albumin. Urine and blood were collected at the time of sacrifice. Serum BUN and urine albumin were increased in FHKO mice but remained closer to normal in those mice treated with clodronate Figure 4. Depletion of $\mathrm{M} \phi$ s did not change the antiapoferritin in circulation (results not shown).
3.4. Macrophage Depletion Reduces Glomerular Pathology in FH-Mediated ICGN. Immune complex deposits in the kidney and histopathological features of ICGN were evaluated at the end of the experiment. Immunofluorescence staining for $\mathrm{C} 3$ and IgG revealed that $\mathrm{M} \phi$ depletion did not modulate the immune complexes in this setting. Representative immunofluorescence staining and histopathology are shown in Figure 5. The primary histopathological feature was of diffuse hypercellularity of the glomerular tufts in $\mathrm{fh}^{-1-}$ mice with CSS was significantly reduced on depletion of $\mathrm{M} \phi$ s.

3.5. Macrophages Modulate Cytokines in FH-Dependent ICGN. Once $\mathrm{M} \phi$ s infiltrate the kidney and are exposed to inflammatory stimuli, they liberate cytokines. We evaluated, by ELISA, plasma circulating levels of TNF $\alpha$ and IL- 6 and the kidney mCSF in FHKO mice treated with saline, apoferritin, or apoferritin and clodronate. The circulating levels of IL-6 were very low in wild-type mice and increased in FHKO mice and were reduced in FHKO mice treated with clodronate (Figure 6). The three cytokines, TNF $\alpha$, IL-6, and mCSF were significantly increased in mice treated with apoferritin compared to saline-treated controls. $\mathrm{M} \phi$ depletion by clodronate significantly limited the rise in cytokines in this setting.

3.6. Macrophages Modulate Profibrotic Molecules and Laminin in FH-Dependent ICGN. Once the kidneys develop a disease, they tend to become fibrotic. $M \phi s$ participate in the fibrotic process by overproducing profibrotic molecules. Real-time PCR revealed a 75\% increase in transforming growth factor-beta1 (TGFbeta1) mRNA expression, a 100\% increase in metalloproteinase-9 (MMP9) expression and $230 \%$ increase in laminin mRNA. In line with the mRNA expression, laminin protein expression is significantly increased in the $\mathrm{fh}^{-/-}$mice with CSS which was reduced in the absence of $\mathrm{M} \phi$ s (Figure 7).

\section{Discussion}

Our recent studies have shown that in the absence of $\mathrm{FH}$, mice develop diffuse proliferative glomerulonephritis (GN) with $\mathrm{F} 4 / 80^{+} \mathrm{M} \phi$ s around sites of ICs [10]. Furthermore, 

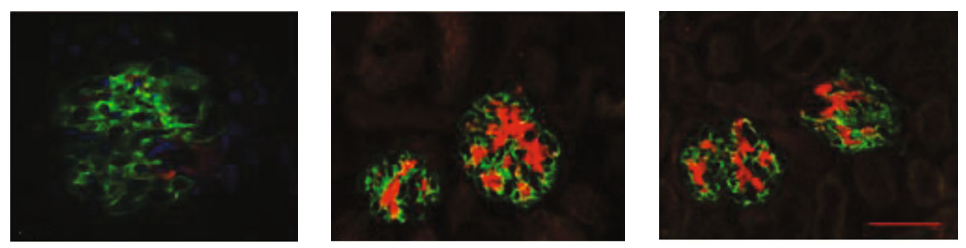

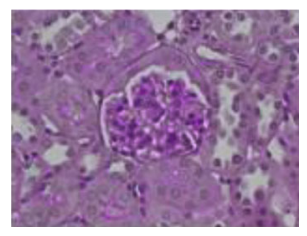

Treatment saline

(a)

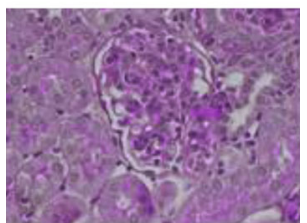

Apoferritin

(b)

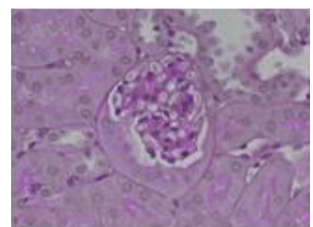

Apoferritin+clodronate

(c)

FIGURE 5: M $\phi$ s depletion reduces glomerular pathology in FH-dependent ICGN. Given are representative images of glomeruli from salinetreated (a), apoferritin-treated (b), and apoferritin+clodronate-treated FH-deficient mice. In (A), the sections are stained with C3 (green) and IgG (red), while (B) show sections stained with PAS. IgG deposits that were increased with apoferritin treatment remained unchanged, while the glomeruli looked closer to normal on macrophage depletion.

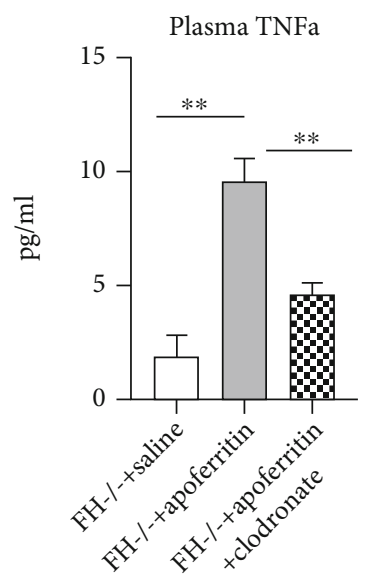

(a)

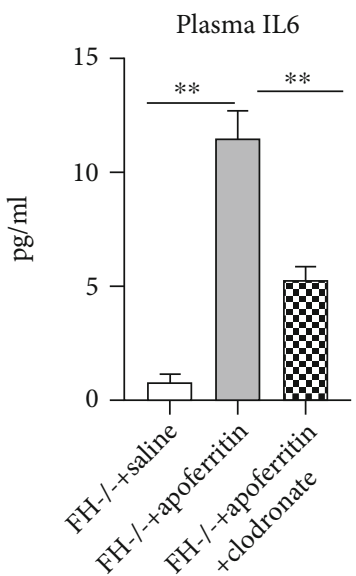

(b)

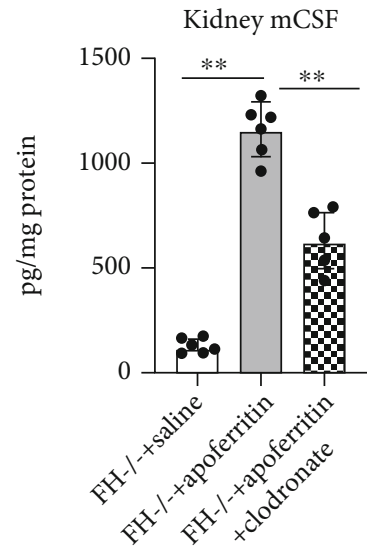

(c)

FIgUre 6: $\mathrm{M} \phi$ s modulate cytokines in FH-dependent ICGN. The plasma and kidneys obtained at the time of sacrificed were stored at $-80^{\circ} \mathrm{C}$ and processed as given in Materials and Methods. The concentrations of plasma TNF $\alpha$ (a), plasma IL-6 (b), and kidney M-CSF (c) were determined by ELISA. Results are representative of multiple experiments. ${ }^{*} P<0.05$, ${ }^{* *} P<0.01$, and ${ }^{* * *} P<0.001$.

our studies demonstrate that $\mathrm{Cd}_{1} 1 \mathrm{~b}^{-1-} \mathrm{fh}^{-1-}$ mice have exacerbated features of glomerulonephritis [26]. Given that the presence of increased inflammatory M1 macrophages and their sustained activation could cause tissue pathology, and their repolarization into the anti-inflammatory phenotype would help in resolution of disease pathology, the current study was undertaken to further understand the role of $\mathrm{M} \phi \mathrm{s}$ in $\mathrm{fh}^{-/-}$mice with ICGN. The increased presence of $\mathrm{M} \phi \mathrm{s}$ in the kidney and the resolution of disease pathology by depletion of macrophages during FH-mediated ICGN demonstrate the critical role of $\mathrm{M} \phi \mathrm{s}$ in this setting, and therefore, they could serve as important therapeutic targets in this setting.

Absence of $\mathrm{FH}$ leads to increased $\mathrm{C} 5 \mathrm{a}$ production. $\mathrm{C} 5 \mathrm{a}$ binds to C5aR1 signaling Mos to the kidney where they add to the inflammatory milieu. In addition, our studies also demonstrated that in this setting of excessive C5a generation, inactivation of C5a appears to limit the disease [10].
Activation of Mos causes them to become polarized, but they remain in a state of continuum [27]. Our results in this study show that glomerular disease in CSS was reduced in $\mathrm{fh}^{-/-}$ mice in which $\mathrm{M} \phi$ s were depleted compared to $\mathrm{fh}^{-/-}$mice without macrophage depletion. Thus, eliminating $\mathrm{M} \phi$ s alleviated ICGN in $\mathrm{fh}^{-/-}$mice reducing the disease pathology. However, $\mathrm{M} \phi$ s are also necessary for the repair process, and since $\mathrm{M} \phi \mathrm{s}$ change states from pro- to antiinflammatory states and vice versa, it will be our future endeavor to understand whether altering the $M \phi$ s landscape without depleting them could alter the disease pathology.

$\mathrm{M} \phi$ s play an important role in the initiation and maintenance of fibrosis and are also involved in the suppression, resolution, and reversal of fibrosis [28]. Our results show increased expression of CD204+ and CD206+ M $\phi$ markers suggesting their increased presence in this setting. Since these $\mathrm{M} \phi$ s are a major source of profibrotic molecules, our results showing increase in TGF $\beta-1$ as well as MMP9 suggest 

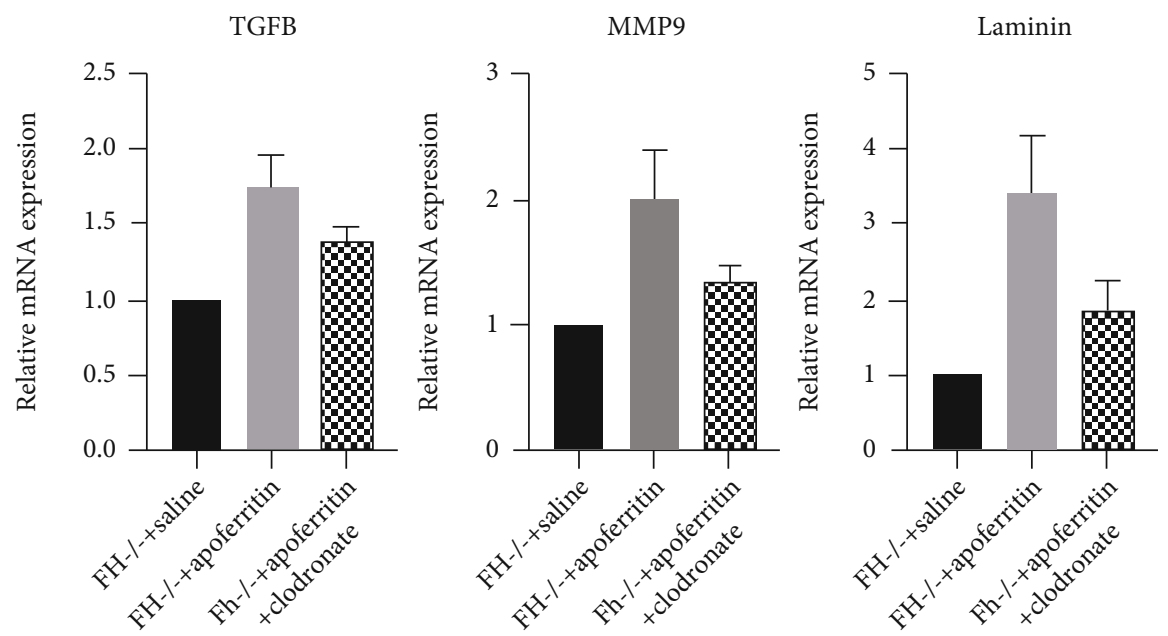

(a)
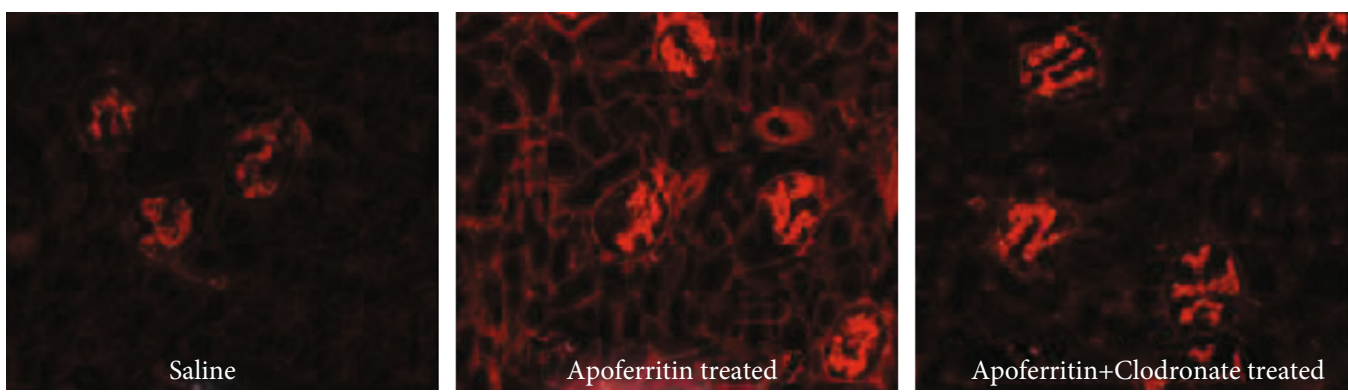

(b)

FIGURE 7: Laminin deposition in FH-deficient kidneys with ICGN is reduced by M $\phi$ s depletion. (a) qRT-PCR for TGFB, MMP9, and laminin from the kidney mRNA isolated from mice after 5 weeks of saline and apoferritin and 3 weeks of clodronate. The expression is normalized to GAPDH ( $n=6$ /group). (b) Representative IF photomicrographs for laminin staining in the kidneys of the same groups of mice. ${ }^{*} P<0.01$.

that these $\mathrm{M} \phi$ s could be contributing both directly and indirectly to fibrotic processes in FH-mediated ICGN through different mechanisms $(1,3,8,44)$. TGF $\beta$-1 can alter fibrosis by cytokine regulation. In addition, TGF $\beta-1$ modulates $\mathrm{C} 3$ and factor $\mathrm{B}$ biosynthesis and expression in monocytes [29], and alteration of complement in the immune cells and kidney cells can facilitate tissue fibrosis [30]. However, the exact interactions between macrophages and complement, the mechanism/s involved, their roles, and tissue compartmentalization need to be deciphered [31]. Our studies showed reduced expression of laminin in $\mathrm{M} \phi$ s-depleted mice compared to $\mathrm{M} \phi$-sufficient mice. To better understand how FH regulates the fibrotic process, we need to identify the specific $\mathrm{M} \phi$ subsets that are present in FH-mediated ICGN and elucidate the contributions of each subset population. These studies are important since complement therapeutics has become a clinical reality. Inhibitors have been and continue to be developed against different targets in the complement cascade that will block the downstream steps in the in the activation pathway(s). Currently, one of the complement inhibitor in clinical use is the anti-C5 antibody, Eculizumab [32]. Therefore, identifying the pathogenic complement components in the disease and supplementing that with regulation of the $\mathrm{M} \phi$ s population is not a trivial point.
In summary, here we studied the role of $\mathrm{M} \phi \mathrm{s}$ in the $\mathrm{FH}$ dependent ICGN model. The results can also be applied in settings of unregulated complement activation with immune complexes such as those observed in different diseases including lupus nephritis, IgA nephropathy, and membranous nephropathy. We show the importance of both complement regulation by $\mathrm{FH}$ and the $\mathrm{M} \phi$ s status in the disease. Thus, pharmacological targeting of the complement cascade and the $\mathrm{M} \phi$ s status in IC-mediated GN has potential clinical relevance.

\section{Data Availability}

All data generated during this study are included in this published article.

\section{Ethical Approval}

All procedures and work on mice in this study were approved by the Institutional Animal Care and Use Committee. The protocol complies with the American Veterinary Medical Association (AVMA) Guidelines for the Euthanasia of Animals, and the ethical principles established by the National 
Institutes of Health Guide for the Care and Use of Laboratory Animals (NIH Publications No. 8523, revised 2011).

\section{Disclosure}

Abstract was presented at the International complement workshop conference: "https://www.sciencedirect.com/ science/article/abs/pii/S0161589018302244?via\%3Dihub."

\section{Conflicts of Interest}

No conflicts of interest, financial or otherwise, are declared by the authors.

\section{Authors' Contributions}

J.J.A and A.J. conceived and designed the research. M.P., S.F., and M.L. performed the experiments. A.C. (pathologist) read the slides. J.J.A and A.J. analyzed the data. J.J.A., A.J., and R.J.Q. interpreted results of experiments, prepared figures, and wrote the manuscript.

\section{Acknowledgments}

This work was supported by NIH R01 DK111222 to JJA and an endowment from Dr. Arthur M. Morris to RJQ.

\section{References}

[1] T. Mijovic, A. Zeitouni, and I. Colmegna, "Autoimmune sensorineural hearing loss: the otology-rheumatology interface," Rheumatology, vol. 52, no. 5, pp. 780-789, 2013.

[2] M. Abongwa, K. E. Baber, R. J. Martin, and A. P. Robertson, "The cholinomimetic morantel as an open channel blocker of the Ascaris suum ACR-16 nAChR," Invertebrate Neuroscience, vol. 16, no. 4, 2016.

[3] A. S. De Vriese, S. Sethi, J. Van Praet, K. A. Nath, and F. C. Fervenza, "Kidney disease caused by dysregulation of the complement alternative pathway: an etiologic approach," Journal of the American Society of Nephrology, vol. 26, no. 12, pp. 29172929, 2015.

[4] R. J. Quigg, "Complement and autoimmune glomerular diseases," Current Directions in Autoimmunity, vol. 7, pp. 165180, 2003.

[5] M. C. Carroll, "The complement system in regulation of adaptive immunity," Nature Immunology, vol. 5, no. 10, pp. 981986, 2004.

[6] M. V. Carroll and R. B. Sim, "Complement in health and disease," Advanced Drug Delivery Reviews, vol. 63, no. 12, pp. 965-975, 2011.

[7] M. Noris and G. Remuzzi, "Overview of complement activation and regulation," Seminars in Nephrology, vol. 33, no. 6, pp. 479-492, 2013.

[8] V. P. Ferreira, A. P. Herbert, C. Cortes et al., "The binding of factor $\mathrm{H}$ to a complex of physiological polyanions and $\mathrm{C} 3 \mathrm{~b}$ on cells is impaired in atypical hemolytic uremic syndrome," Journal of Immunology, vol. 182, no. 11, pp. 7009-7018, 2009.

[9] J. J. Alexander, M. C. Pickering, M. Haas, I. Osawe, and R. J. Quigg, "Complement factor h limits immune complex deposition and prevents inflammation and scarring in glomeruli of mice with chronic serum sickness," Journal of the American Society of Nephrology, vol. 16, no. 1, pp. 52-57, 2005.

[10] J. J. Alexander, L. Chaves, A. Chang, and R. J. Quigg, “The C5a receptor has a key role in immune complex glomerulonephritis in complement factor H-deficient mice," Kidney International, vol. 82, no. 9, pp. 961-968, 2012.

[11] C. V. Jakubzick, G. J. Randolph, and P. M. Henson, "Monocyte differentiation and antigen-presenting functions," Nature Reviews. Immunology, vol. 17, no. 6, pp. 349-362, 2017.

[12] Q. Cao, Y. Wang, and D. C. Harris, "Macrophage heterogeneity, phenotypes, and roles in renal fibrosis," Kidney International. Supplement, vol. 4, no. 1, pp. 16-19, 2014.

[13] Q. Cao, Y. Wang, X. M. Wang et al., "Renal F4/80+ CD11c+ mononuclear phagocytes display phenotypic and functional characteristics of macrophages in health and in adriamycin nephropathy," Journal of the American Society of Nephrology, vol. 26, no. 2, pp. 349-363, 2015.

[14] K. Kitamoto, Y. Machida, J. Uchida et al., "Effects of liposome clodronate on renal leukocyte populations and renal fibrosis in murine obstructive nephropathy," Journal of Pharmacological Sciences, vol. 111, no. 3, pp. 285-292, 2009.

[15] S. A. Sung, S. K. Jo, W. Y. Cho, N. H. Won, and H. K. Kim, "Reduction of renal fibrosis as a result of liposome encapsulated clodronate induced macrophage depletion after unilateral ureteral obstruction in rats," Nephron. Experimental Nephrology, vol. 105, no. 1, pp. e1-e9, 2007.

[16] J. C. Frith, J. Monkkonen, G. M. Blackburn, R. G. Russell, and M. J. Rogers, "Clodronate and liposome-encapsulated clodronate are metabolized to a toxic ATP analog, adenosine 5'-(beta, gamma-dichloromethylene) triphosphate, by mammalian cells in vitro," Journal of Bone and Mineral Research: the Official Journal of the American Society for Bone and Mineral Research, vol. 12, no. 9, pp. 1358-1367, 1997.

[17] J. J. Alexander, O. G. Aneziokoro, A. Chang et al., "Distinct and separable roles of the complement system in factor $\mathrm{H}$ deficient bone marrow chimeric mice with immune complex disease," Journal of the American Society of Nephrology, vol. 17, no. 5, pp. 1354-1361, 2006.

[18] J. J. Alexander, L. D. Chaves, A. Chang, S. Dighe, A. Jacob, and R. J. Quigg, "Abrogation of immune complex glomerulonephritis by native carboxypeptidase and pharmacological antagonism of the C5a receptor," Cellular \& Molecular Immunology, vol. 13, no. 5, pp. 651-657, 2016.

[19] R. J. Quigg, A. Lim, M. Haas, J. J. Alexander, C. He, and M. C. Carroll, "Immune complex glomerulonephritis in C4- and C3deficient mice," Kidney International, vol. 53, no. 2, pp. 320 330, 1998.

[20] L. Bao, M. Haas, S. A. Boackle et al., "Transgenic expression of a soluble complement inhibitor protects against renal disease and promotes survival in MRL/lpr mice," Journal of Immunology, vol. 168, no. 7, pp. 3601-3607, 2002.

[21] S. Gordon, "Alternative activation of macrophages," Nature Reviews. Immunology, vol. 3, no. 1, pp. 23-35, 2003.

[22] S. Gordon and P. R. Taylor, "Monocyte and macrophage heterogeneity," Nature Reviews. Immunology, vol. 5, no. 12, pp. 953-964, 2005.

[23] A. Mantovani, S. K. Biswas, M. R. Galdiero, A. Sica, and M. Locati, "Macrophage plasticity and polarization in tissue repair and remodelling," The Journal of Pathology, vol. 229, no. 2, pp. 176-185, 2013. 
[24] S. D. Ricardo, H. van Goor, and A. A. Eddy, "Macrophage diversity in renal injury and repair," The Journal of Clinical Investigation, vol. 118, no. 11, pp. 3522-3530, 2008.

[25] D. D. Kim, T. Miwa, Y. Kimura, R. A. Schwendener, C. M. van Lookeren, and W. C. Song, "Deficiency of decay-accelerating factor and complement receptor 1-related gene/protein y on murine platelets leads to complement-dependent clearance by the macrophage phagocytic receptor CRIg," Blood, vol. 112, no. 4, pp. 1109-1119, 2008.

[26] J. J. Alexander, L. D. Chaves, A. Chang, A. Jacob, M. Ritchie, and R. J. Quigg, "CD11b is protective in complementmediated immune complex glomerulonephritis," Kidney International, vol. 87, no. 5, pp. 930-939, 2015.

[27] P. J. Murray, "Macrophage polarization," Annual Review of Physiology, vol. 79, no. 1, pp. 541-566, 2017.

[28] M. Hesketh, K. B. Sahin, Z. E. West, and R. Z. Murray, "Macrophage phenotypes regulate scar formation and chronic wound healing," International Journal of Molecular Sciences, vol. 18, no. 7, p. 1545, 2017.

[29] A. K. Hogasen, K. Hestdal, K. Hogasen, and T. G. Abrahamsen, "Transforming growth factor $\beta$ modulates $\mathrm{C} 3$ and factor $\mathrm{B}$ biosynthesis and complement receptor 3 expression in cultured human monocytes," Journal of Leukocyte Biology, vol. 57, no. 2, pp. 287-296, 1995.

[30] J. Cui, X. Wu, Y. Song, Y. Chen, and J. Wan, "Complement C3 exacerbates renal interstitial fibrosis by facilitating the M1 macrophage phenotype in a mouse model of unilateral ureteral obstruction," American Journal of Physiology. Renal Physiology, vol. 317, no. 5, pp. F1171-F1182, 2019.

[31] D. Portilla and S. Xavier, "Role of intracellular complement activation in kidney fibrosis," British Journal of Pharmacology, vol. 178, no. 14, pp. 2880-2891, 2021.

[32] M. Le Quintrec, A. Lionet, C. Kandel et al., "Eculizumab for treatment of rapidly progressive C3 glomerulopathy," American Journal of Kidney Diseases, vol. 65, no. 3, pp. 484-489, 2015. 